Research Paper

\title{
Anti-Cervical Cancer Role of Matrine, Oxymatrine and Sophora Flavescens Alkaloid Gels and its Mechanism
}

\author{
Yu Jie Zhou, Ya Jie Guo, Xiao Li Yang, Zhou Luo Ou ${ }^{\varpi}$ \\ Key Laboratory of Breast Cancer in Shanghai, Breast Cancer Institute, Fudan University Shanghai Cancer Center, Department of Oncology, Shanghai Medical \\ College, Fudan University, Shanghai 200032, China. \\ $\triangle$ Corresponding author: Zhou Luo Ou, Department of Oncology, Breast Cancer Institute, Fudan University Shanghai Cancer Center, 270 Dong An Road, \\ Shanghai 200032, People's Republic of China. Tel: +86 21 6417 5590. Fax: +86 216417 4774. Email: zlou@shmu.edu.cn \\ (C) Ivyspring International Publisher. This is an open access article distributed under the terms of the Creative Commons Attribution (CC BY-NC) license \\ (https://creativecommons.org/licenses/by-nc/4.0/). See http://ivyspring.com/terms for full terms and conditions.
}

Received: 2017.08.17; Accepted: 2018.01.19; Published: 2018.04.06

\begin{abstract}
Background: Cervical cancer is one of the leading severe malignancies throughout the world. Sophra flavescens alkaloid (SFA) gels, a compound Traditional Chinese Medicine, has been clinically used in China for many years. Its individual active ingredients are matrine and oxymatrine, which has been showed that they can restrain primary tumorigenesis, while the underlying molecular mechanisms of SFA gels in cervical cancer cells remain unclear.

Methods: To detect the effect of SFA gels and its active ingredients, CCK-8 assay and colony assay were used on cervical cancer cells proliferation. Transwell assay was used to detect cancer cell migration. Apoptosis and cell cycle arrest were used to detect whether SFA gels effect the cervical cancer cells proliferation. Western blot was used to detect whether SFA gels regulate the cervical cancer cells via the suppression of AKT/mTOR signaling pathway.

Results: SFA gels can restrain cervical cancer cell proliferation, inhibit metastasis, induce cell cycle arrest in G2/M phase, induce cellular apoptosis through stimulation of Bax and E-cadherin, and suppression of Bcl-2, cyclin A, MMP2. Further study shows that SFA gels may regulate the cervical cancer cells via the suppression of AKT/mTOR signaling pathway.

Conclusions: SFA gels, like its active ingredients, can restrain cervical cancer cells proliferation, suppress cervical cancer cell migration, induce the apoptosis and cell cycle arrest in cervical cancer cells. SFA gels may be a potential anti-tumor therapeutic agent for treating cervical cancer.
\end{abstract}

Key words: Sophora flavescens alkaloid gels; Matrine; Oxymatrine; Cervical cancer; AKT; mTOR

\section{Introduction}

Cervical cancer remains a major cause of cancer-related mortality worldwide and often diagnosed at an early clinical stage [1]. Chemotherapy and radiotherapy are the common therapeutic interventions for cervical cancer either as primary treatment, adjuvant, or neoadjuvant therapy [2].

Sophora flavescens alkaloid (SFA) gels, also known as Kushen, a compound Traditional Chinese Medicine, has been clinically used in China for many years, and there are many clinical reports demonstrating its anti-cancer effect. These reports include using sophora flavescens alkaloid gels to treat gastric cancer, liver cancer, lung cancer, breast cancer, ovarian cancer, colorectal cancer and other cancer types [3, 4]. Chinese scholars have found that SFA gels can use in the treatment of aerobic vaginitis, cervical erosion and vaginal fungal infection [5]. Its effective ingredients mainly include matrine and oxymatrine and increasing evidences have demonstrated that matrine and oxymatrine exert its anticancer effect on various cancer types, such as prostate cancer, lung cancer, acute myeloid leukemia and so on. For matrine, it can inhibit cancer cells by restraining cell proliferation, inhibiting cells apoptosis and cell cycle arrest, suppressing invasion and regulating singling pathways [6-9]. Besides, some studies indicated that 
oxymatrine may prevent pulmonary hypertension through its anti-proliferative, anti-inflammatory, and antioxidant effects [10]. Oxymatrine can induce apoptotic cell death of human pancreatic cancer [11] and prostate cancer [12] Some research found that oxymatrine combining HMGB1 may be more effective in the treatment of human synovial sarcoma and oxymatrine may induce autophagy [13]. These studies suggested that matrine and oxymatrine may be effective in anti-tumor activities. However, the effect of Sophora flavescens alkaloid gels and its mechanism on cervical cancer cells have not yet been evaluated.

The protein kinase $\mathrm{B} /$ mammalian target of rapamycin (AKT/mTOR) is an important intracellular signaling pathway. PI3K/AKT/mTOR signaling pathway has already been reported that related with cell activities like, proliferation, migration and invasion [14, 15]. We suspect to find some relationship between Sophora flavescens alkaloid gels and AKT $/ \mathrm{mTOR}$ signaling pathway.

In this study, we showed that Sophora flavescens alkaloid gels can restrain cervical cancer cell proliferation, migration and invasion, block cell cycle in G2/M phase and induce cellular apoptosis through stimulation of Bax and E-cadherin, and suppression of Bcl-2, Cyclin A, MMP2. Further study illustrated that the antitumor effects of the SFA gels in the cervical cancer cells may induce through the AKT/mTOR signaling pathway. SFA gels may be a potential anti-tumor therapeutic agent for treating cervical cancer and might have broad applications to improve clinical outcome.

\section{Materials and Methods}

\section{Cell lines and cell culture}

The cervical cancer cell lines SiHa and C33A were given by Professor Gong Yang. Ca Ski were obtained from Cell Bank of Chinese Sciences Academy. SiHa and C33A cells were cultured in Dulbecco's Modified Eagle's Medium (DMEM, Shanghai Yuanpei Company) and Ca Ski and HK-2 were cultured in RPMI 1640 (Shanghai Yuanpei Company), supplemented with $10 \%$ fetal bovine serum (FBS) (Gibco), $100 \mathrm{U} / \mathrm{mL}$ penicillin, and 100 $\mathrm{U} / \mathrm{mL}$ streptomycin. The cells were incubated at 37 in a humidified $5 \% \quad \mathrm{CO}_{2}$ atmosphere. HK-2 (human kidney-2), a proximal tubular epithelial cell line derived from normal human kidney, was used as a normal control cell line in this study.

\section{Drugs and reagents}

SFA gels was provided by Guiyang Xin Tian Pharmaceutical Co., Ltd. (lot number JG141002; Guizhou, China). Matrine (Lot NoMKBS8661V) and Oxymatrine (Lot No 011M1378V) were purchased from Sigma. Antibodies against AKT (4691, 1:1000), Phospho-AKT (4060P, 1:1000), E-Cadherin (3195,1:1000), MMP2 (13132, 1:1000) and mTOR Substrates Sampler kit (CST 9862), which includes Phospho-mTOR, mTOR, were purchased from Cell Signaling Technology. Antibodies against Bcl-2 (ab32124, 1:1000), Bax (ab32503, 1:1000), Cyclin A (ab181591, 1:2000), CDK2 (ab32147, 1:1000) were from Abcam.

\section{Growth inhibition assay}

The growth inhibition assay of SFA gels was measured by Cell Counting Kit-8(CCK-8) (Dojindo Laboratories, Kumamoto, Japan). Briefly, $1 \times 10^{3}$ cells per well were plated in 96-well plates and treated with SFA gels, matrine and oxymatrine at an array of concentrations for 48 hours. Then, each well added 10 $\mu \mathrm{L}$ CCK-8 solution and incubated at $37^{\circ} \mathrm{C}$ for 2 hours. $\mathrm{IC}_{50}$ was calculated by GraphPad 6 .

\section{Colony formation assay}

$1 \times 10^{3}$ cells were seeded in six-well plates at a single cell density and SFA gels were added on the second day, then cells were incubated for 2 weeks. The colonies with more than 50 cells were counted after staining with crystal violet (Solarbio).

\section{Cell cycle analysis}

Cell cycle status was detected by flow cytometry and analyzed by Multicycle AV (for windows, version 320) software. Briefly, cells were treated with SFA gels, matrine and oxymatrine at various concentrations for $48 \mathrm{~h}$, and then harvested, washed twice with $1 \times$ PBS, and re-suspended in $200 \mu \mathrm{L}$ of $1 \times$ PBS. The cells were fixed in $4 \mathrm{~mL}$ of ice-cold $75 \%$ ethanol at $4^{\circ} \mathrm{C}$ overnight and stained with $200 \mu \mathrm{L}$ of propidium iodide $(50 \mu \mathrm{g} / \mathrm{mL}$, Sigma-Aldrich) and 20 $\mu \mathrm{L}$ of RNase $(1 \mathrm{mg} / \mathrm{mL}$, Sigma-Aldrich) to remove RNA in a $37^{\circ} \mathrm{C}$ water bath for 15 to 20 minutes. The cells were then analyzed by flow cytometry (Cytomics FC 500 MPL, Beckman Coulter). All the tests were performed three times.

\section{Cell apoptosis analysis}

To detect apoptosis, cells were incubated with SFA gels, matrine and oxymatrine at different concentrations for $48 \mathrm{~h}$. The cells were harvested, washed twice with cold $1 \times$ PBS, and re-suspended in $200 \mu \mathrm{L}$ binding buffer at density of $1 \times 10^{5}$ cells $/ \mathrm{ml}$. The cells were then stained with $5 \mu \mathrm{L}$ Annexin- $\mathrm{V}$ and PI (BD Biosciences) for 15 min in dark condition at room temperature and subjected to analysis by flow cytometry (Cytomics FC 500 MPL, Beckman Coulter). Cells that are considered viable are FITC Annexin V and PI negative, cells that are in early apoptosis are FITC Annexin V positive and PI negative, while cells 
that are in late apoptosis or already dead are both FITC Annexin V and PI positive. All the tests were performed three times.

\section{Cell migration assay}

Migration assays were performed using transwell insert polycarbonate membranes (8 $\mathrm{Mm}$ pore size, BD, Franklin Lakes, NJ, USA). To test cell migration, $1 \times 10^{5}$ cells of $\mathrm{SiHa}$ or $\mathrm{C} 33 \mathrm{~A}$ treated with SFA gels, matrine and oxymatrine at different concentrations and their corresponding controls were added in upper chamber and $600 \mu \mathrm{L}$ DMEM with $10 \%$ fetal bovine serum was added to the bottom at $37^{\circ} \mathrm{C}$ for 24 hours. Nonmigratory cells on the upper membrane surface were removed, and migratory cells attached to the bottom surface of the membrane were then fixed in $100 \%$ methanol for 30 minutes and stained with crystal violet for 10 minutes. All the tests were performed three times.

\section{Western blot analysis}

Western blot analysis was performed to determine the expression levels of various proteins in cells. Cells were treated with SFA gels at different concentrations for $48 \mathrm{~h}$. Cells were harvested, washed with cold $1 \times$ PBS, and lysed with RIPA lysis buffer (Beyotime) for $30 \mathrm{~min}$ on ice, then centrifuged at $10,000 \mathrm{~g}$ for $15 \mathrm{~min}$ at $4{ }^{\circ} \mathrm{C}$. The total protein concentration was detected by BCA protein assay kit (Beyotime). Equal amounts ( $40 \mu \mathrm{g}$ per load) of protein samples were subjected to SDS-PAGE electrophoresis and transferred on to Polyvinylidene Fluoride (PVDF) membranes (Millipore). The blots were blocked in 5\% non-fat milk, and incubated with primary antibodies overnight, followed by incubation with secondary antibodies conjugated with horseradish peroxidase (HRP). The protein bands were developed with the chemiluminescent reagents (Millipore).

\section{Statistical analysis}

Statistical analysis was performed using Statistical Package for the Social Sciences (SPSS) software Version 22.0 for Windows (SPSS Inc., Chicago, IL, USA). Analysis of variance and Student's t-test were used to determine the statistical significance of differences between experimental groups in vitro. Values of $\mathrm{P}<0.05$ were considered statistically significant. Graphs were created with GraphPad Prism 5 (GraphPad Software, Inc., La Jolla, CA, USA).

\section{Results}

SFA gels and its active ingredients can restrain cervical cancer cells proliferation

To examine the effect of SFA gels on proliferation of cervical cancer, Siha, C33A, Ca Ski and HK-2 cells were treated with increasing concentrations of SFA gels and its effective ingredients matrine and oxymatrine for two days, and cell proliferation was detected by CCK-8 assay. As shown in Figure $1 \mathrm{~A}, 1 \mathrm{~B}$ and $1 \mathrm{C}$, the percentage of inhibition in the four cell lines was dramatically increased with a dose-dependent character when treated with SFA gels, and this phenomenon was also found in matrine as well as oxymatrine.

Next, we used colony formation assays to further measure SFA gels' growth inhibitory effects. After 14 days of incubation, the results clearly showed that both colony numbers and the size of the colonies in Siha were reduced by SFA gels (Fig 1D). However, the results of $\mathrm{C} 33 \mathrm{~A}$ and $\mathrm{Ca}$ ski were not as obvious as the cell SiHa (Fig S1 and S2).

These data further confirmed that SFA gels inhibit cell growth and colony formation in cervical cancer cells.

\section{SFA gels and its active ingredients suppress cervical cancer cell migration}

We further assessed whether SFA gels have any effect on the migration of cervical cancer cells. Transwell assay was used to evaluate the cervical cell migration. Cells were treated under different concentrations of SFA gels, matrine and oxymatrine for 48 hours to measure cell migration. According to the growth inhibitory effect, $0.75 \mathrm{mg} / \mathrm{ml}$ of SFA gels was treated to cervical cancer cells as low concentration, and $1.5 \mathrm{mg} / \mathrm{ml}$ was treated as high concentration. While cells treated under concentrations at $1 \mathrm{mg} / \mathrm{ml}$ and $2 \mathrm{mg} / \mathrm{ml}$ of matrine and oxymatrine were recorded as low concentration and high concentration. After 24 hours, the number of the cervical cells that migrated through the membrane in the bottom chamber was significantly reduced when cells were treated with SFA gels. The same trends were observed in cells treated with matrine and oxymatrine. We found that, compared with control groups, SFA gels decreased the migration speed of cervical cancer cells (Fig 2A, 2B 2C, 2D).

Further, we evaluated the expression level of cell adhesion-associated proteins by Western blot, including matrix metalloproteinase 2 (MMP2) and E-cadherin. The results show that after treated with SFA gels, the level of E-cadherin was increased and MMP2 was decreased in cervical cancer cell, and the expression levels were in a concentration-dependent manner (Fig 2E).

Therefore, SFA gels effectively impair the ability of migration in cervical cancer cells by reducing the expression of MMP2 and increasing the expression of E-cadherin. 


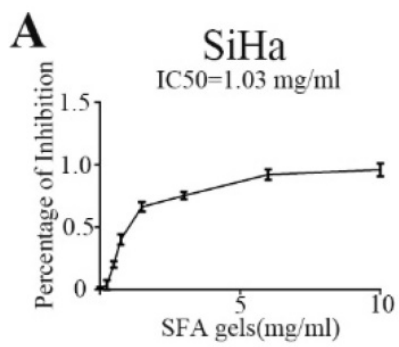

Ca ski
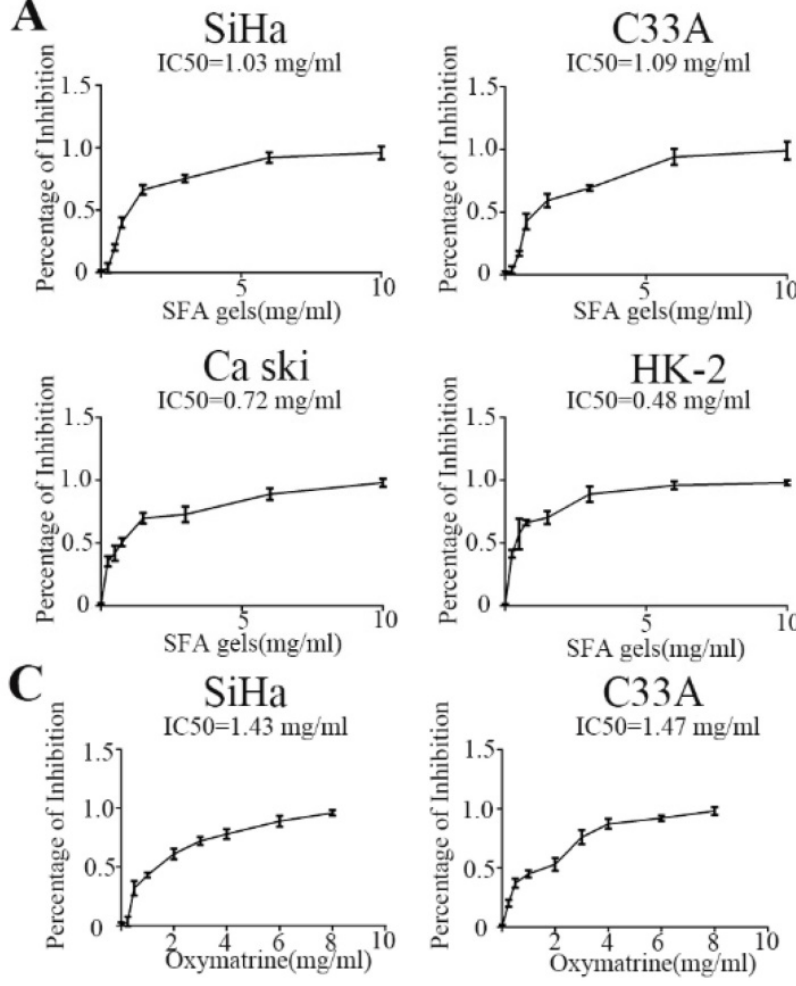

HK-2

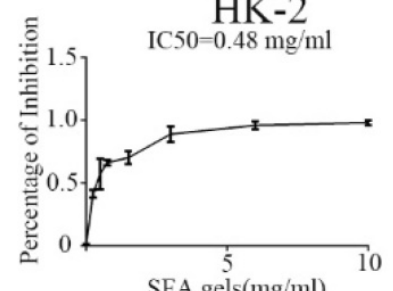

$\mathrm{C} 33 \mathrm{~A}$
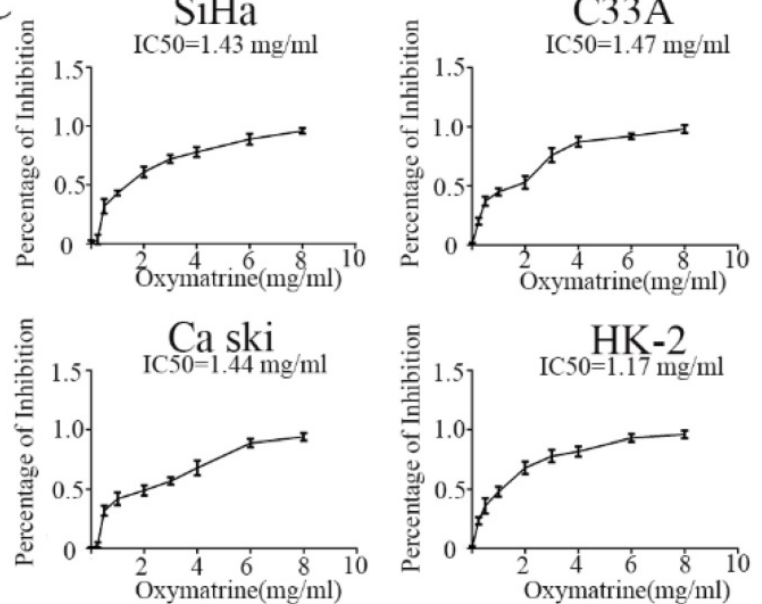
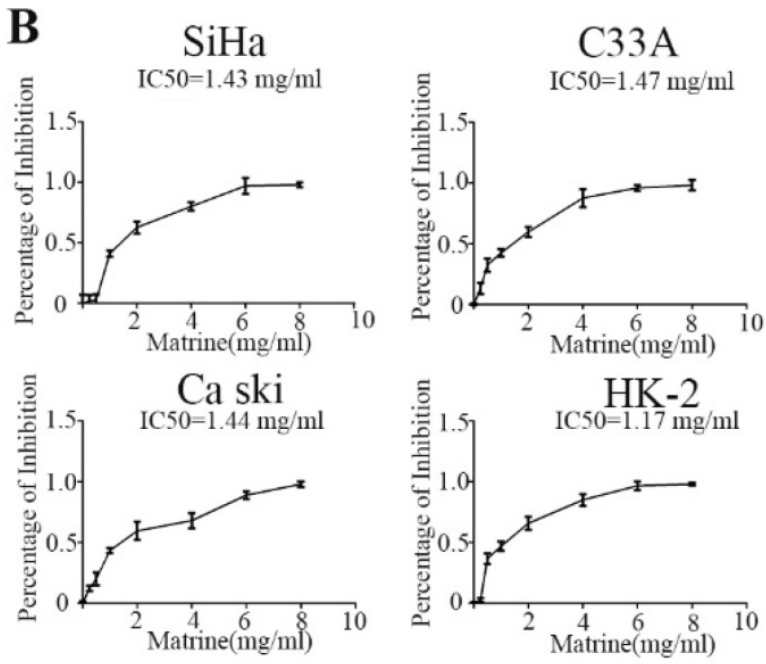

D

Figure 1. Sophora flavescens alkaloid gels and its active ingredients can restrain cervical cells proliferation. (A), (B) and (C) Growth inhibition assay of SiHa, $\mathrm{C} 33 \mathrm{~A}$, Caski and HK-2 was detected by CCK8 assay under different concentrations of Sophora flavescens alkaloid gels (A), matrine (B) and oxymatrine (C) for 48 hours. (D) colony assay of $\mathrm{SiHa}$ was used to further measure Sophora flavescens alkaloid gels' growth inhibitory effect.

\section{SFA gels induce the apoptosis and cell cycle arrest in cervical cancer cells}

Matrine and oxymatrine have been reported to induce apoptosis. This fact interested us to investigate whether SFA gels have the same effect. Firstly, cervical cancer cells were double stained by Annexin $\mathrm{V}$ and PI and detected by flow cytometry to analyze the apoptotic effects after treated by SFA gels for 48 hours. Cells that are considered viable are FITC Annexin V and PI negative, cells that are in early apoptosis are FITC Annexin V positive and PI negative, while cells that are in late apoptosis or already dead are both FITC Annexin V and PI positive. The total apoptotic cells were made up with the early apoptotic cells and the late apoptotic cells. As is shown in Fig 3A and 3B, the percentage of total apoptotic cells increased with the increasing concentrations of SFA gels, which indicated that SFA gels stimulate cervical cancer cells in a dose-dependent manner. Further, the result of
Western Blot illustrated that the pro-apoptotic protein Bax was increased and anti-apoptotic protein Bcl-2 was decreased after SFA gels' treatment (Fig 3E).

To investigate whether SFA gels inhibit cell proliferation through blocking cell cycle, we used flow cytometry to detect the cell cycle after 48 hours treatment of SFA gels. As is showed in Fig 3C and 3D, the percentage of cells at G0-G1 and S phase were decreased, while the percentage at G2-M phase was increased compared with cells in control group. Besides, we analyzed the major proteins associated with cell cycle progression by Western blotting. The results showed that the levels of cyclin A and CDK2, which play an important role at G2 phase, were decreased after 48 hours' SFA gels treatment. And the proteins reduced in a dose-dependent way (Fig 3F).

Taken all these together, we provided strong evidence that SFA gels induced anti-cancer activities by inducing cell apoptosis and blocking cell cycle progression. 


\section{SFA gels inhibit the cervical cancer cell activities through AKT/mTOR signaling pathways}

The AKT/mTOR pathway is an important intracellular signaling pathway in regulating various progressions in cancer cell. SFA gels' effective ingredients matrine has already been reported that disrupts AKT/mTOR signal pathways. To determine the potential mechanism of the anti-cancer effects of

A

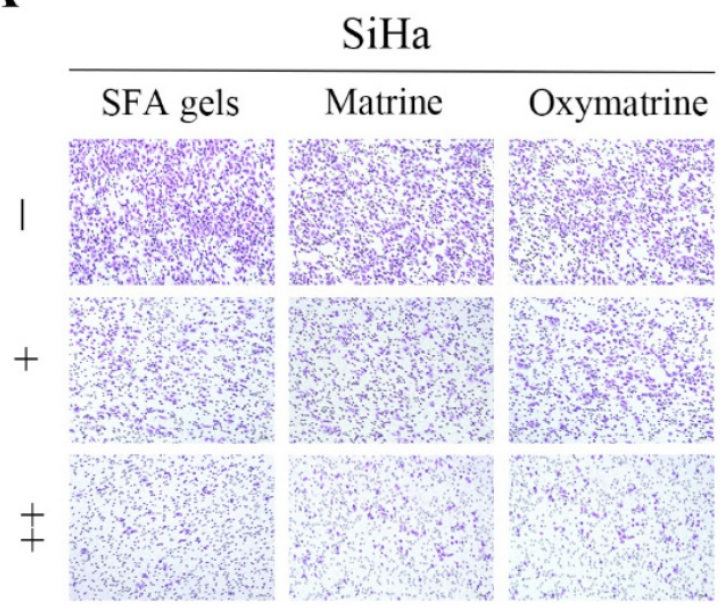

C
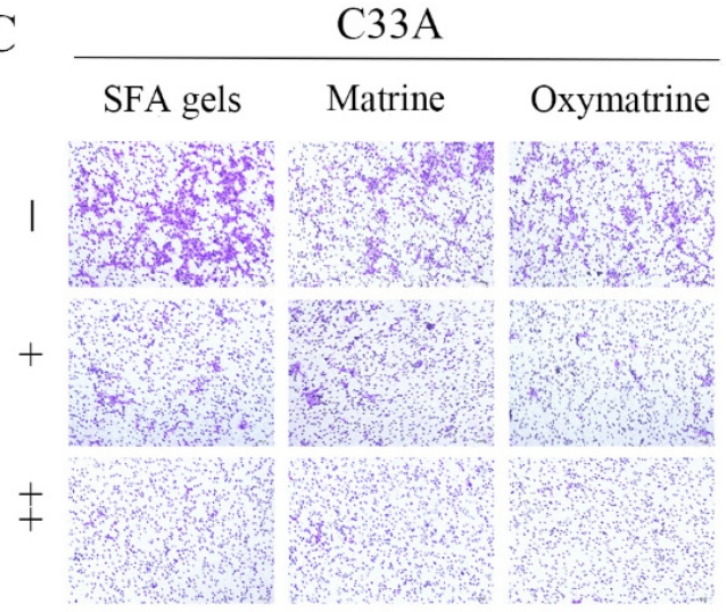

B

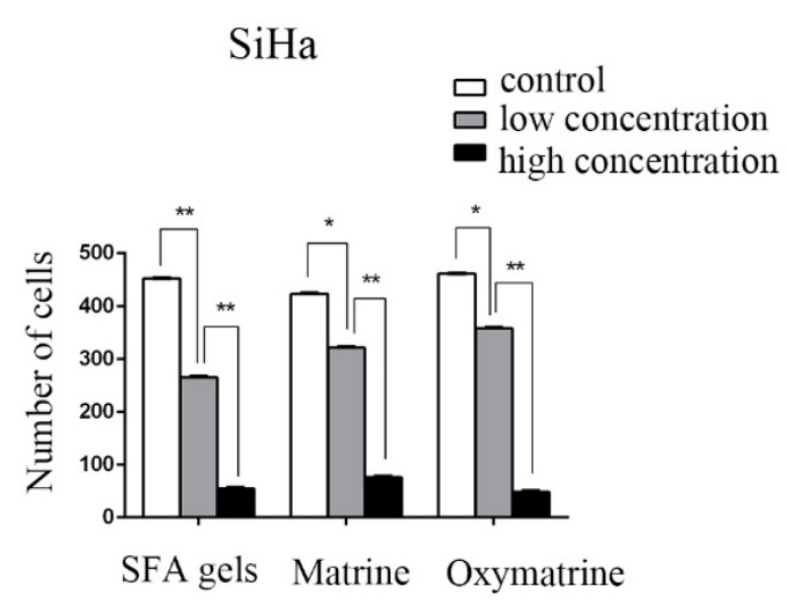

D

SFA gels, we detected this pathway. The level of phosphorylated AKT and mTOR was evaluated. As is showed in Fig 4, SFA gels significantly suppressed the phosphorylation of AKT, mTOR in a dose-dependent manner in SiHa and C33A. These findings showed that SFA gels negatively regulate the phosphorylation of $\mathrm{AKT} / \mathrm{mTOR}$ signaling pathway to involve cervical cancer cell anti-cancer effect.

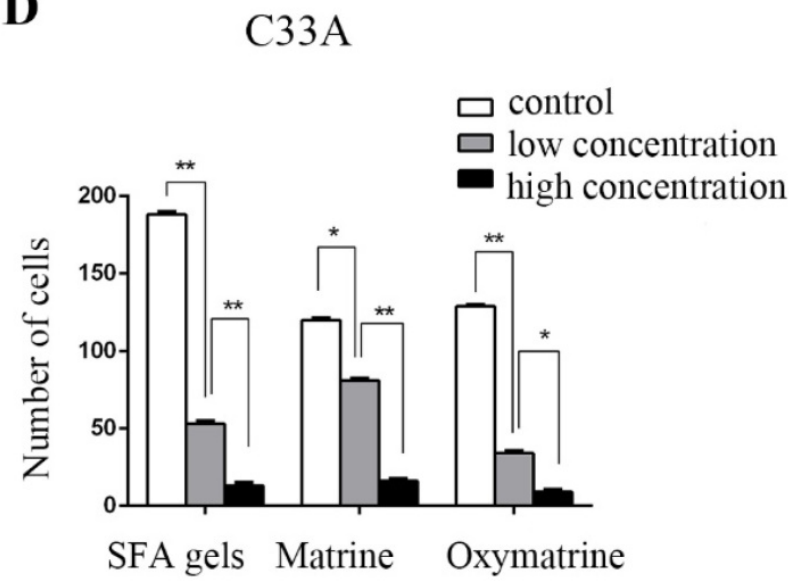

$\mathbf{E}$

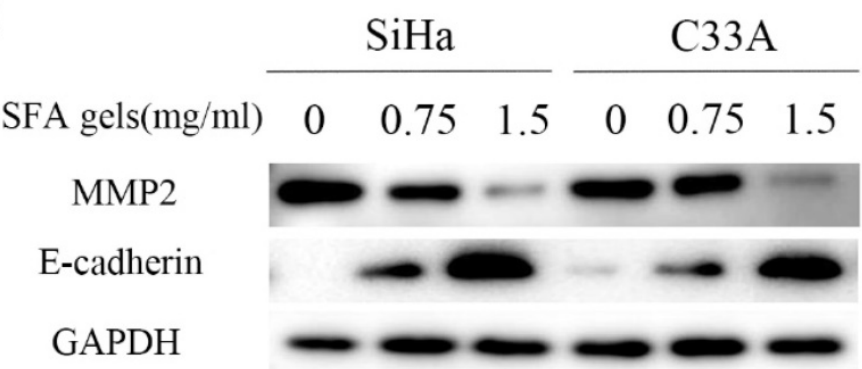

Figure 2. Sophora flavescens alkaloid gels suppress cervical cancer cell migration. (A), (C) Cell migration was detected by the transwell assay. (B), (D) Columns represent quantification of invaded cells, ${ }^{* P}<0.05$, $* * P<0.01$. Error bars $=95 \%$ Cis. (E) Expression of cell adhesion-associated protein E-cadherin and MMP2 were detected by Western Blot. 
A
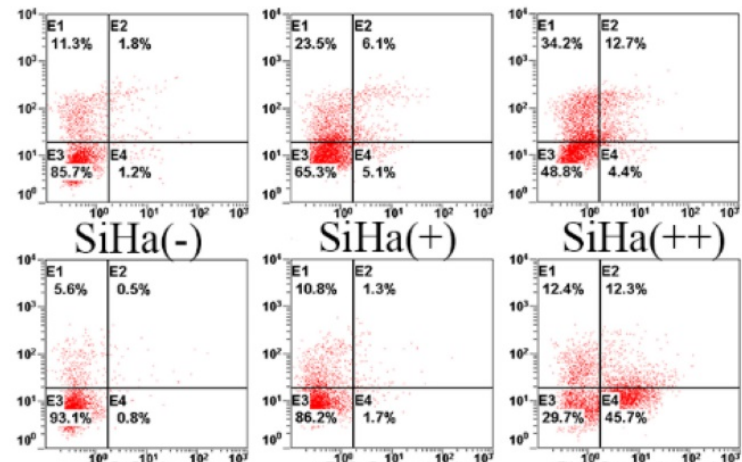

$\mathrm{SiHa}(+)$

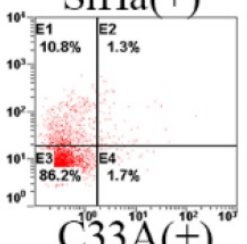

$\mathrm{SiH}^{10} \mathrm{Hit}(++)^{10}$

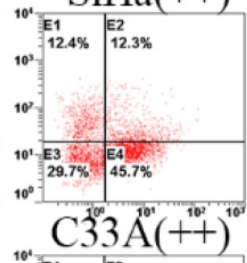

$\mathrm{Pi}$
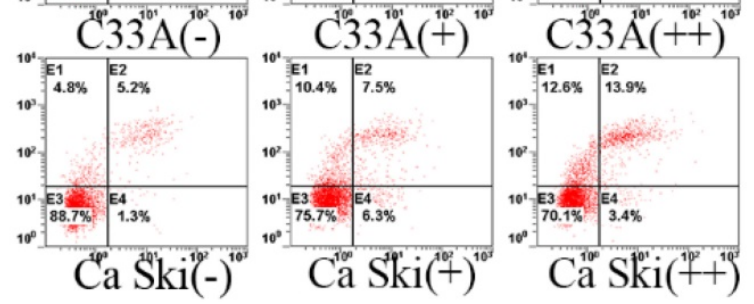

$$
\text { Annexin V-FITC }
$$

C
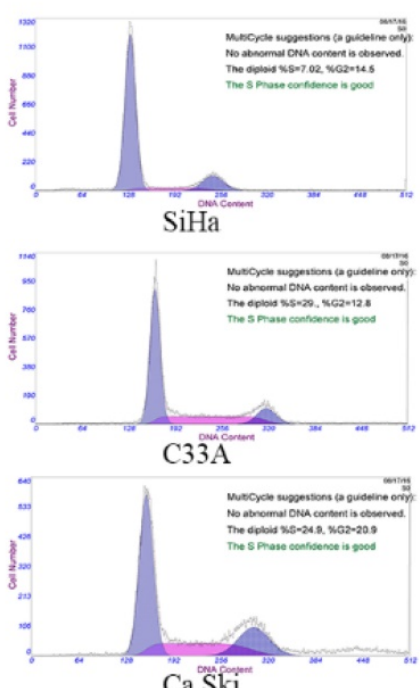

E

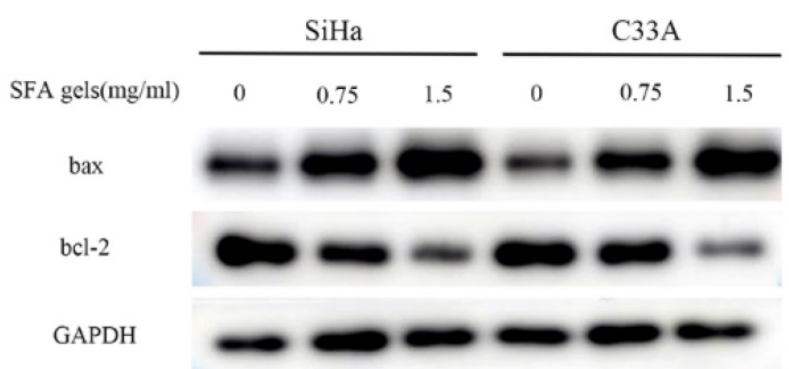

B

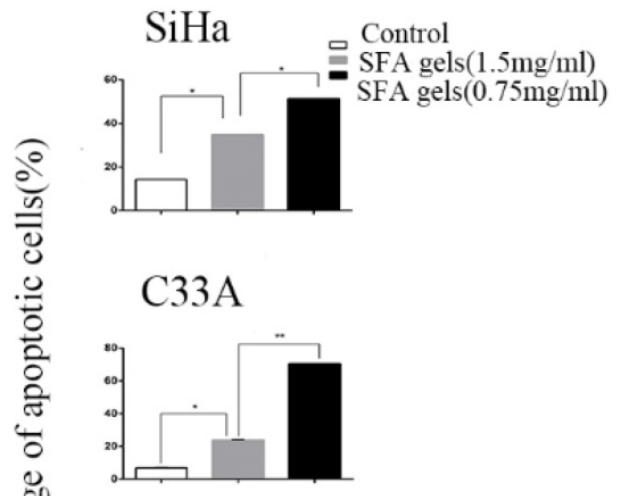

Ca Ski

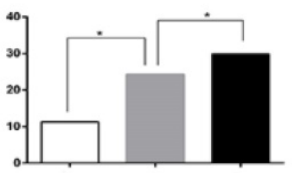

D

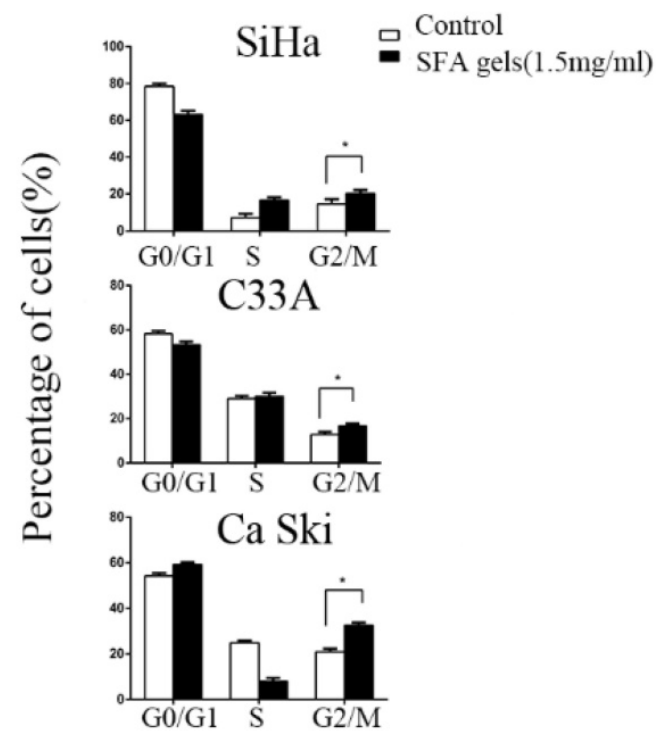

F

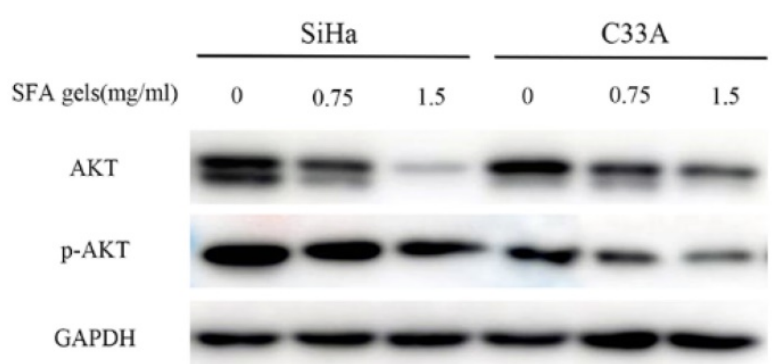

Figure 3. Sophora flavescens alkaloid gels induces the apoptosis and cell cycle arrest in cervical cancer cells. (A) Flow cytometry apoptosis analysis was performed in cultured Siha, C33A and Ca ski cell lines harvested after 2 days of treatment with different concentration of SFA gels, matrine and oxymatrine. (B) Quantification of apoptosis for the cell lines. $* P<0.05, * * P<0.01$. Columns are means of three independent experiments, bars are SD. (C) Cell cycle analysis was performed using flow cytometry by staining with propidium iodide. (D) Columns represent quantification of cell phase. (E) and (F) Western Blot analysis of apoptosis-associated and cell cycle regulatory proteins. 


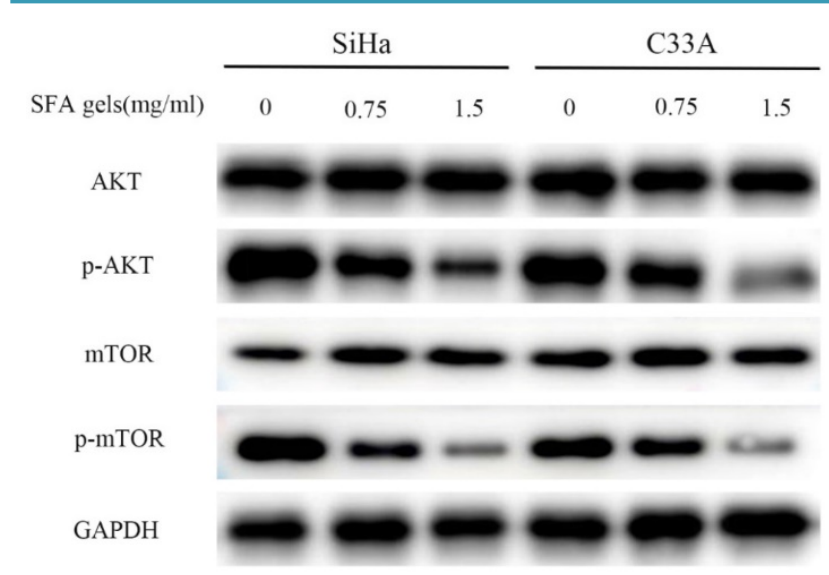

Figure 4. Sophora flavescens alkaloid gels inhibit the cervical cancer cell anti-cancer activities through AKT/mTOR signaling pathways. Expression of the components of the AKT/mTOR pathways were detected by Western blot in both $\mathrm{SiHa}$ and $\mathrm{C} 33 \mathrm{~A}$ cell lines.

\section{Discussion}

For cervical cancer, surgery, chemotherapy and radiotherapy are still the common therapies. However, these therapies may bring patients side-effect. In recent years, traditional Chinese medicine (TCM) has gained more and more acceptance worldwide and increasing evidence have shown that TCM also have the anti-cancer effect [16].

Sophora flavescens alkaloid, also called Kushen, has been widely used in clinical trials, and there are many clinical reports demonstrating its anti-cancer effect [4]. These reports include using sophora flavescens alkaloid to treat gastric cancer, liver cancer, lung cancer, breast cancer, ovarian cancer, colorectal cancer and other cancer types [3].The efficacy of Kushen has been reported either alone or in cooperation with chemotherapeutic or radiotherapeutic treatments in the cancer patients. Bao et al reported a systematic review showed positive evidence of Kushen for bone cancer pain. Kushen appeared to be able to improve total pain relief and quality of life, and seems to have beneficial effects on reduction of side effects in patients with bone cancer pain compared with radiotherapy or bisphosphonates [17]. Sun et al confirmed that Kushen plus transcatheter arterial chemoembolization (TACE) is more superior to TACE alone for unresectable hepatocellular carcinoma (UHCC) patients [18].

In this study, we detected the SFA gels. Its effective ingredients were matrine and oxymatrine, which have already been reported that can exert the anti-cancer effect on many cancer types.HK-2, performed in this study, is a proximal tubular cell (PTC) line derived from normal human kidney. We used HK-2 as a normal control cell line in this study. Our results showed that SFA gels and its ingredients can restrain cervical cells proliferation, detected by CCK8 assay and colony assay. Through the transwell assay, the SFA gels can suppress cervical cancer cell migration. Western blot showed that MMP2 protein was reduced and E-cadherin protein was increased, which can lead to enhanced cellular invasion as compared to control cells.

Further data showed that SFA gels' treatment increased the pro-apoptotic protein Bax, but decreased the anti-apoptotic protein Bcl-2. The cyclin $\mathrm{A}$ and $\mathrm{CDK} 2$ protein play a critical role in the transition of S/G2 phase. Our data showed that the levels of cyclin A and CDK2 were also reduced after SFA gels treatment, consistent with the reduction in $S$ phase and G2/M arrest in flow cytometry analysis. Further mechanistic studies showed that AKT/mTOR pathways were activated through reduced the expression of $\mathrm{p}$-AKT and $\mathrm{p}$-mTOR.

In recent studies, many mechanisms about matrine and oxymatrine have been reported related to their anti-cancer effect. However, the compound medicine has no reported so far. Our works aim to detect the mechanism of SFA gels antitumor effect and to provide SFA gels broad applications for anti-inflammatory treatment, preventing precancerous lesions, improving cervical cancer clinical outcome. SFA gels may be a potential anti-tumor therapeutic agent for treating cervical cancer.

\section{Abbreviations}

SFA gels: Sophra flavescens alkaloid gels; AKT: Protein Kinase B; mTOR: mammalian Target of Rapamycin; BCA: Bicinchoninic acid; BSA: Bovine Serum Albumin; CCK-8: Cell counting kit-8; DMEM: Dulbecco's Modified Eagle's Medium; ECL: Enhanced chemiluminescence reagent.

\section{Supplementary Material}

Supplementary figures.

http://www.jcancer.org/v09p1357s1.pdf

\section{Competing Interests}

The authors have declared that no competing interest exists.

\section{References}

1. Siegel RL, Miller KD, Jemal A. Cancer statistics, 2018. CA Cancer J Clin. 2018; 68:7-30.

2. Wright JD, Chen L, Tergas AI, et al. Population-level trends in relative survival for cervical cancer. Am J Obstet Gynecol. 2015; 213 (670e): 1-7.

3. He X, Fang J, Huang L, et al. Sophora flavescens Ait.: Traditional usage, phytochemistry and pharmacology of an important traditional Chinese medicine. J Ethnopharmacol. 2015; 172:10-29.

4. Wang W, You RL, Qin WJ, et al. Anti-tumor activities of active ingredients in Compound Kushen Injection. Acta Pharmacol Sin. 2015; 36:676-9.

5. Wang X, Li J, Hu Y, et al. Effect of Sophora flavescens alkaloid on aerobic vaginitis in gel form for local treatment. J Tradit Chin Med. 2017; 37:314-20. 
6. Huang $\mathrm{H}, \mathrm{Du} \mathrm{T}, \mathrm{Xu} \mathrm{G}$, et al. Matrine suppresses invasion of castration-resistant prostate cancer cells by downregulating MMP-2/9 via NF-kappaB signaling pathway. Int J Oncol. 2017; 50:640-8.

7. Wang Z, Zhang J, Wang Y, et al. Matrine, a novel autophagy inhibitor, blocks trafficking and the proteolytic activation of lysosomal proteases. Carcinogenesis. 2013; 34:128-38.

8. Wu J, Hu G, Dong $\mathrm{Y}$, et al. Matrine induces Akt/mTOR signalling inhibition-mediated autophagy and apoptosis in acute myeloid leukaemia cells. J Cell Mol Med. 2017; 21:1171-81.

9. Zhou $\mathrm{H}, \mathrm{Xu} \mathrm{M}, \mathrm{Gao} \mathrm{Y}$, et al. Matrine induces caspase-independent program cell death in hepatocellular carcinoma through bid-mediated nuclear translocation of apoptosis inducing factor. Mol Cancer. 2014; 13:59.

10. Zhang B, Niu W, Xu D, et al. Oxymatrine prevents hypoxia- and monocrotaline-induced pulmonary hypertension in rats. Free Radic Biol Med. 2014; 69:198-207.

11. Ling $Q, X u X$, Wei $X$, et al. Oxymatrine induces human pancreatic cancer PANC-1 cells apoptosis via regulating expression of Bcl-2 and IAP families, and releasing of cytochrome c. J Exp Clin Cancer Res. 2011; 30:66.

12. Wu C, Huang W, Guo Y, et al. Oxymatrine inhibits the proliferation of prostate cancer cells in vitro and in vivo. Mol Med Rep. 2015; 11:4129-34.

13. Cai $Y, X u$ P, Yang L, et al. HMGB1-mediated autophagy decreases sensitivity to oxymatrine in SW982 human synovial sarcoma cells. Sci Rep. 2016; 6:37845.

14. Yang N, Qu YJ, Cheng Y, et al. Endoplasmic reticulum stress regulates proliferation, migration and invasion of human ovarian cancer SKOV3 cells through PI3K/AKT/mTOR signaling pathway. Cancer Biomark. 2017; 19:263-9.

15. Zhong JT, Yu J, Wang HJ, et al. Effects of endoplasmic reticulum stress on the autophagy, apoptosis, and chemotherapy resistance of human breast cancer cells by regulating the PI3K/AKT/mTOR signaling pathway. Tumour Biol. 2017; 39:1010428317697562.

16. Hsiao $\mathrm{WL}$, Liu L. The role of traditional Chinese herbal medicines in cancer therapy--from TCM theory to mechanistic insights. Planta Med. 2010; 76:1118-31.

17. Yanju B, Yang L, Hua B, et al. A systematic review and meta-analysis on the use of traditional Chinese medicine compound kushen injection for bone cancer pain. Support Care Cancer. 2014; 22:825-36.

18. Sun $\mathrm{Q}, \mathrm{Ma} \mathrm{W}, \mathrm{Gao} \mathrm{Y}$, et al. Meta-analysis: therapeutic effect of transcatheter arterial chemoembolization combined with compound kushen injection in hepatocellular carcinoma. Afr J Tradit Complement Altern Med. 2012; 9:178-88. 